\title{
On Chinglish in Chinese-English Translation and Its Countermeasures: Taking Translation of Modern Chinese Prose as an Example
}

\author{
Pianpian Huang \\ Department of College English, Zhejiang Yuexiu University, Shaoxing, China \\ Email: 37783302@qq.com
}

How to cite this paper: Huang, P.P. (2021) On Chinglish in Chinese-English Translation and Its Countermeasures: Taking Translation of Modern Chinese Prose as an Example. Open Access Library Journal, 8: e7472. https://doi.org/10.4236/oalib.1107472

Received: April 29, 2021

Accepted: June 6, 2021

Published: June 9, 2021

Copyright $\odot 2021$ by author(s) and Open Access Library Inc.

This work is licensed under the Creative Commons Attribution International License (CC BY 4.0).

http://creativecommons.org/licenses/by/4.0/ (c) (i) Open Access

\begin{abstract}
Prose, containing colorful Chinese culture, is one of the most important treasures of Chinese literature. And in order to spread it abroad, the translation of it plays an indispensable role, because the good English version helps foreigners to understand more about Chinese culture. However, due to the different environments as well as thinking models between English and Chinese, translators are affected by the Negative Transfer, and couldn't successfully transform their thinking patterns and thus bring about various kinds of Chinglish while in the practice of Chinese-English translation of prose. The subject of this paper is dozens of prose translations carefully completed by students' cooperation in groups. On the basis that the translations are basically free of grammatical errors, the author explores Chinglish in those translations from the perspective of Negative Transfer, focusing on the main categories and causes of Chinglish at lexical and syntactic levels, and putting forward relative countermeasures.
\end{abstract}

\section{Subject Areas}

Linguistics

\section{Keywords}

Chinglish, Negative Transfer, Prose Translations, Translation

Countermeasures

\section{1. 概述}

随着中国改革开放的不断深入, 大量的国际友人对中国文化产生了浓厚 的兴趣, 散文是中国文化的一个重要方面。然而散文翻译中的 Chinglish 现象 
(中式英语)层出不穷, 连精通英语的人也难以避免, 既影响了中国的形象, 也严重阻碍了中国文化的传播。本文的研究对象为本校汉语言文学专业的大 二学生, 为期两个学期, 所学的专业与英语相结合, 通过小组合作, 多次修 改后所得的数十篇散文译文。本文从母语负迁移的角度, 针对这些译文, 研 究其中式英语的主要形式和成因, 以此希望译者译出规范的译文, 最终进行 有效地文化交流。

\section{2. 母语负迁移和Chinglish (中式英语)}

20 世纪中期, 以 Fries 与 Lado 为首的学者开始对语言迁移进行研究, 他 们指出语言迁移是二语习得时母语对外语目标语所产生的影响。到了 20 世纪 末, 学者对语言迁移这一概念有了更为客观的认识。 Rod Ellis 提出: “It is difficult to quantify the extent of transfer in the different language levels” [1]. 翻 译领域也不可避免的受其影响。语言迁移根据其产生的影响可以分为两类: 正迁移(产生积极影响)和负迁移(产生消极影响)。李伯利认为负迁移也叫 “抑 制性迁移”, 是指一种学习对另一种学习产生阻碍作用 [2]。比如: 在刚学英 语语法时, 由于受汉语语法的影响, 有人会用汉语的语法套在英语的语法运 用当中, 如 She go study every morning 就是负迁移。翻译时, 汉语母语对译 者多年来的影响会自然的产生干扰作用, 使其将汉语知识移入译文当中, 由 此便产生了不地道的英文一一中式英语(Chinglish)。

李文中提出中国式英语是指中国的英语学习和使用者由于受母语干扰和 影响, 硬套汉语规则和习惯, 在英语交际中出现的不合规范英语或不合英语 文化习惯的畸形英语[3]。比如: 中国人有句常用的问候语 “你干什么去啊? 吃过饭了吗? ” 有些人就把它用到英语中去了, 有了 “Where are you going? Have you eaten yet?” 这就是非常明显的中式英语。再比如: “你有两下子” 翻译为 “You have two down son”。这里要强调的是: “中式英语” 与 “中 国英语(China English)” 不是同一概念。葛传槊提出中国英语是一种以规范英 语为核心, 表达中国社会文化的特有事物, 主要通过音译、借译及语义再生 等手段进入英语交际, 具有中国特点的词汇、句式和语篇的英语 [4]。比如: 廉政建设——construct clean politics; 经济特区——special economic zone; 四 个现代化一一four modernization; 一箭双雕——One shot, two hawks 等。因 此, 与 “中式英语” 不一样, “中国英语” 是符合英语习惯的, 标准和规范的, 是可接受的。本文研究的是前者—— “中式英语” , 既不符合英语标准和规 范的表达方式。

\section{3. 从词汇方面分析散文英译中的Chinglish现象及其成因}

本文以学生合作完成的中国现代散文英译为研究对象, 接下来直接从词 汇方面开始探讨 Chinglish 现象的四种主要表现形式及其成因。

\section{1. 词汇方面的 Chinglish 现象}

\subsection{1. 同义堆叠}

巴金的《木匠老陈》中有这么一句: 有人说他去吃粮当了兵。被译为: 
Someone said he had become a soldier eating the national grain. 但是, 实际上 句子中的旧时用法 “吃粮” 就是 “当兵” 的意思, 译为英文时保留 “当兵” 这一含义即可, 所以句子可以译为: Someone said he had gone soldiering.

\subsection{2. 词汇多余}

Pinkham 曾提出 “Unnecessary words are the hallmark of Chinglish” [5]。 也就是说, 中式英语的一大特点就是词汇, 诸如名词、动词或形容词等的多 余, 现举例具体说明:

许地山的散文《落花生》中倒数第三段中的词语 “鲜红嫩绿的颜色” 被 误译为 “brilliant red and light green colors”, 这里的名词 “红” 和 “绿” 就 是多余, “brilliant”一词就涵盖了这两种颜色, 所以译为 “brilliant colors” 即可。

同一文中第一段的 “我们几姊弟和几个小Y头都很欢喜” 被译为 “Our several sisters and brothers, and also our servant girls were all excited”。“姊弟” 一词在下文中将涉及, 所以以防累赘, 合并这两个名词, 改译为 “children” 更好, 形容词 “several” 则可以省去。更好的译文 “We children and our servant girls were all excited” 。

\subsection{3. 搭配不当}

英语与汉语的搭配原则截然不同。如果翻译时不加思考, 按照汉语习惯 搭配了, 这样在英语中肯定是不恰当的。

胡适《差不多先生传》第四段的 “凡事只要差不多, 就好了。何必太精 明呢？”后面的问句被译为: Why be too shrewd? “shrewd”一词有 “精明的, 精于盘算的, 有眼光的” 意思。但是根据上下文得知, 文中要表达的是 “精 确, 准确” 之意, 所以更为恰当的译文为: What's the use of being precise and accurate?

\subsection{4. 重复指称}

英语中有一个指导原则, 那就是: 应避免不必要的重复。但在汉语中重 复表达十分常见, 起着强调的作用。于是就出现了这种类型的 Chinglish 现象。

矛盾《白杨礼赞》第一句中的“白杨树是在是不平凡的, 我赞美白杨树!”, 被译为: The white poplars are not ordinary, so let me pay compliments to those poplars. 句中的 “poplars” 在相邻句皆出现了, 即为重复指称。后一个 “those poplars” 用代词 “them” 来代替。译文后半句改为: so let me pay compliments to them. 对于此类中式英语, 可以用代词或关系代词来代替上文相同的事物, 这样更符合英语的行文规范。

\section{2. 词汇方面 Chinglish 现象的成因}

根据语言负迁移理论, 译者把汉语中的各类词性想当然地译入英文是不 自觉的, 因为在汉语中这些词确实传达必要的意义。另外, 译者受汉语用语 习惯的影响, 忽视了英语本身的规则, 也是造成中式英语的原因。William Strunk 提出: “Vigorous writing is concise. A sentence should contain no un- 
necessary words, a paragraph no unnecessary sentences, for the same reason that a drawing should have no unnecessary lines and a machine no unnecessary parts” [6]。英语用词简洁, 句子和段落里不应有多余的词和句子, 但在汉语 中适当的重复有增强节奏或加强语义的作用。再次, 不同的历史文化背景使 得英汉两种语言形成了各自固定的表达和搭配方式, 故译者翻译时汉语潜在 的影响就自然地融入到英译中, 由此就产生了 Chinglish 现象。

\section{4. 从句法方面分析散文英译中的Chinglish现象及其成因}

\section{1. 句法方面的 Chinglish 现象}

句法研究是对句子结构和句子各成分之间关系的研究, 以中国现代散文 英译为例, 句法方面的 Chinglish 现象最典型的有以下几类:

\subsection{1. 垂悬修饰语不当}

翻译时, 句首的短语如果与后面的句子逻辑关系混乱时, 这类不符合英 语文法的短语就称之为不当的垂悬修饰语。

叶圣陶的《我坐上了木船》第五段中作者提到他托找关系, 设法去买船 票或是飞机票, 困难重重, 最后 “跑了不知多少趟, 总算有眉目了”, 引号 中的句子被误译为: After making countless visits, signs of a positive outcome finally appear. 句子中, 从句中的短语 “making countless visits” 的主语根据 文章意思判断应该是 “I”。但是根据句子结构来看, 从句主语跟后面主句的 主语应为一致, 也就是 “signs of a positive outcome” , 这样从句的意思就成 为 “积极结果的迹象(或眉目)跑了不知多少趟了”, 就不符合逻辑。所以我 们要把这个垂悬修饰语改明确, 把句子改写为 “After I have made countless visits, signs of a positive outcome finally appear”。

\subsection{2. 短语或从句的位置不当}

朱自清《背影》第五段中的 “他和我走到车上, 将橘子一股脑儿放在我 的皮大衣上……说: ‘我走了, 到那边来信!'”译为: He put all the tangerines on my coat ... and said that he must go and asked me not to forget to write to him after getting on the train with him. 短语“ after boarding the train with him” 本是和放橘子的动作是前后承接的关系, 放在句尾就曲解了愿意, 变成 “上 火车” 之后就 “写信” 的意思了。为了避免歧义, 句子应改为: After getting on the train with him, he put all the tangerines on my coat ... and said that he must go and asked me not to forget to write to him. 所以译者应该反复检查句 子各成分之间的关系, 使译文准确又连贯。

\subsection{3. 平行结构不当}

朱自清《匀匀》首句 “燕子去了, 有再来的时候; 杨柳枯了, 有再青的 时候; 桃花谢了, 有再开的时候。” 被译为: Swallows go away, they will come back again; Willows wither, they will turn green again; Peach blossoms fade, they will flower again. 中文和译文皆为平行结构。但是在正规的英文当中, 逗 号连接的皆是不完整的句子, 然而译文中的三个逗号, 连接的前后句都是完 
整的主谓结构的句子, 这就不符合英文用语规范了。所以应在 “swallows”

“willows” 和 “peach blossoms” 三个词语前分别加个 “If” , 变为三句从句。 三句条件状语从句并列, 作为排比句, 这样才符合英语规范。

\section{2. 句法方面 Chinglish 现象的成因}

句法方面出现的 Chinglish 现象通常是由于译者对句子之间的关系没有 理顺, 按照汉语思维翻译出来的后果, 也就是受到了母语负迁移的干扰。句 法结构上, 英文是从属关系, 而中文则是并列结构。也就是说英语句子按照 语法关系组织起来, 是形合语言; 汉语句子则按逻辑关系组合, 是意合语言。 因而英语句子间的连接形式明显、严谨、丰富, 句子结构组合紧密细致, 汉 语句子各成分之间的关系要靠理解来推断出, 从形式上无直接显现, 简练易 懂。中文中常常用浓缩的句式使句子简明[7]。所以如果在英译时, 译者忽视 了英语重形合的特点, 比如英译时短语和从句的位置摆放不当, 这样就会曲 解原意; 要是不注意英语形式上的统一, 则会造成平行结构使用不当的错误。

\section{5. 解决散文英译中Chinglish问题的主要策略}

\section{1. 词汇方面 Chinglish 现象的解决策略}

汉英翻译时, 对有些词汇的表达不确定时, 译者容易凭字典中的 “对应 词” 死译, 这样明显体现了母语的负迁移。接下来就从英汉词汇方面提出解 决 Chinglish 现象的主要策略。

\subsection{1. 用语更贴近英语习惯}

郭沫若的《墓》最后一段中的 “啊, 死了的我昨日的尸骸”, 被译为: Oh, my yesterday's dead self。在此, 为了烘托散文诗的格调, 更适合翻译为: Oh, ye remains of my yesterday's dead self. 其中 ye 是英语的古语, 意为 “你”。

\subsection{2. 熟记成语和习语}

胡适《不要抛弃学问》第二段中的 “从今以后, 你们可以依自己的心愿 去自由研究了”, 被译为 From now on you are free to do researches according to your own will. 这里 “做自己喜爱或感兴趣的事” 就可以用一个英语成语 “follow one's bent”, 所以更地道的译文应为: From now on you are free to follow your own bent to do researches.

朱自清《多多》第二段中的 “八千多日子已经从我手中溜去” , 被简洁 地译为 more than eight thousand days have already passed. 这样并无不可, 但 是表现不出这个意境。这里可以用一个英语习语 “slip away through one's fingers” 来取代 “pass”一词。

所以译者在平时要加强输入, 多记和熟记英语中的成语和习语, 从而译 出符合英语约定俗成的译文。

\subsection{3. 联系语境}

译者在翻译时不能脱离上下文下笔, 否则也会产生 Chinglish 现象。

巴金的《朋友》第二段中 “在朋友面前我只感到惭愧”, 被译为: I can't 
help feeling ashamed before my friends. 按照字面意思, “惭愧” 意为 “差愧”, 即 ashamed。但是通读上下文, “惭愧” 在文中的意思应为 “不好意思” , 所以译为 “ill at ease” 或者 “embarrassed” 更为恰当。

\section{2. 句法方面 Chinglish 现象的解决策略}

英语和汉语的句法结构区别也有不少, 蒋坚松对此做过详细比较, 提出 两者的主要区别在于: 汉语重意合, 英语重形合; 汉语重人称, 英语重物称; 汉语多动态, 英语多静态; 汉语的展开性和英语的浓缩性等等 [8]。接下来就 从英语和汉语句法上的这些差异方面提出解决 Chinglish 现象的主要策略。

\subsection{1. 汉语意合句可译为英语形合句}

重意合的汉语使用动词的频繁比英语高, 但无词形变化, 甚至不怎么用 关联词。而英语重形合, 其中一大特点是有谓语和非谓语动词之分, 而且常 使用不定式等非谓语形式来表明句子各部分之间的逻辑关系。

胡适的《差不多先生传》第八段中 “他从从容容地走到火车站, 迟了两 分钟, 火车已经开走了”, 被译为 He got to the railway station leisurely, and he was late for two minutes, so the train had already gone. 汉语中连续使用了三个 动词, 来突出差不多先生的特点和其后果。译文稍显零散、拖沓, 用法不是 很规范。可以对其进行重组, 修改为: He got to the railway station leisurely only to find the train had already gone, because he was late for two minutes. 修改后 的句子连接更加紧密, 而且用了 “only to find” 这一动词不定式作结果状语, 比之前更严谨和有序，更符合英语规范。

\subsection{2. 汉语的人称主语转换成英语的物称主语}

汉语主语常用人称，英语则主语为物称倾向。

郁达夫《故都的秋》第三段中 “每年到了秋天, 总要想起陶然亭的芦 花…… , 被译为 Each autumn I would think of the reed catkins of Peiping's Ranting Tao...这句译文平平无奇, 更佳的译文为: The arrival of each autumn would put me in mind of the reed catkins of Peiping's Ranting Tao...汉语的人 称主语 I 转换成了英语的物称主语 The arrival of each autumn, 用法更贴近英 语用语习惯，表达更顺畅。

\subsection{3. 汉语较高级的句法单位可转换成英语较低级的句法单位}

汉、英民族思维存在着差异, 由此汉、英句式也具有展开性和浓缩性的 差异。也就是：相对来说，英语喜欢用较低级的句法单位。

朱自清《背影》第一段中的 “到徐州见着父亲, 看见满院狼藉的东西, 又想起了祖母, 不禁簌籁地留下眼泪” , 被译为: When I met my father in Xuzhou, I saw all the mess in the courtyard, couldn't help thinking of my grandma, and therefore burst into tears. 译文直接套用了汉语的开展性思维, 比较拖沓、啰嗦。改译为: When I met my father in Xuzhou, the sight of all the mess in the courtyard and the thought of my grandma started tears streaming down. 改译后的句子, 由原来主句中三个动词连接的并列三句, 浓缩为由两 
个主语和一个动词组成的一句, 即把汉语中较为高级的句法单位改译成了英 语中较低级的句法单位, 以更符合英语的表达习惯。

\subsection{4. 汉语动态句可译为英语静态句}

较之汉语, 英语更注重静态描写。故汉译英时, 要突出英语的静态特征。 冰心 《我的父母之乡》末段有一句 “他们都动员我回去看看, 我何尝不 想呢？”句中的 “我何尝不想呢” 译为：Why don't I want to do so?这里用了 直译, 动词短语 “want to do so” 的使用使得译句表意啰嗦。为了意思表达更 清晰和紧凑, 可采用静态句型, 改译为: Yes, I am more than eager for that.

\section{3. 译者应注意的其他要点}

首先, 译者要了解原文意思之后再动笔; 译完初稿后, 反复检查, 㽎酌 英文表达，避免中式英语。

其次, 译者的语言功底要扎实, 对上文所提的英语和汉语的词汇和句法 差异牢记于心，可减少 Chinglish 现象的产生。

再次, 注意平时勤积累, 以精通语言为目的。通过各种途径如：上课、 看书、看电影和听电台等去大量接触地道的英语, 以免翻译遇到障碍时总是 不自觉地求助于母语, 造成语言负迁移, 产生中式英语。

最后, 还要扩大知识面, 这样译者翻译起来才会更加得心应手。

\section{6. 结语}

散文是中国文学的瑰宝, 承载了灿烂的中国文化, 要推动散文的海外传 播, 翻译起着不可替代的作用。汉英翻译时, 对于词汇使用译者要更贴近英 语用语习惯; 要联系上下文语境, 切勿望词生 “译” ; 要注意积累, 多记英 语成语和习语。在句法运用方面，译者切勿机械照搬汉语句子，把握其整体 意思之后，根据英语的句法习惯灵活处理、并对不同的句式进行不同的转换。 当然, 由于作者的研究范围欠广, 还有别的 Chinglish 现象还有待发现, 也还 有其它避免中式英语的对策还有待提出。但是在了解以上中式英语的主要形 式、产生的原因和对策后，翻译时可大大减少中式英语的产生。同时，也至 关重要的是译者的综合素质要提高, 这样才可以在中国现代散文英译中, 译 出更地道的英文, 由此将中国现代散文推向世界, 让世界更了解中国文化, 促进中外交流。

\section{Conflicts of Interest}

The author declares no conflicts of interest regarding the publication of this paper.

\section{References}

[1] Ellis, R. (1994) The Study of Second Language Acquisition. Oxford University Press, Oxford.

[2] 李伯利. 语言的负迁移现象与中国式英语的产生[J]. 重庆工商大学学报, 2005(1): 129-132. 
[3] 李文中. 中国英语和中国式英语[J]. 外语教学与研究, 1993(4): 20-26.

[4] 葛传槊. 漫谈由汉译英问题 $[\mathrm{M}] / /$ 翻译理论与翻译技巧论文集. 北京: 中国对外翻 译出版公司, 1982.

[5] Joan, P. (2003) The Translator's Guide to Chinglish. Foreign Language Teaching and Research Press, Beijing.

[6] Strunk Jr., W. (1979) The Elements of Style. Penguin Books, London.

[7] 陈定安. 英汉比较与翻译 [M]. 北京: 中国对外翻译出版公司, 1998.

[8] 蒋坚松. 英汉对比与汉译英研究[M]. 长沙: 湖南人民出版社, 2009.

\section{Appendix (Abstract \& Keywords in Chinese)}

\section{汉英翻译实践中的 Chinglish 现象及对策研究——以中国现代散文的英译为}

例

摘要: 散文是中国文学的瑰宝, 承载了灿烂的中国文化。要推动散文的 海外传播, 翻译起着不可或缺的作用, 因为好的英译版本能让外国友人更多 地了解中国文化。但是由于中西方思维模式和语言环境的不同, 在散文汉英 翻译实践过程中, 译者会受到母语负迁移的干扰, 不能成功地进行思维模式 的转换, 出现不同程度的 Chinglish 现象, 也就是中式英语。本文的研究对象 为学生小组合作、精心完成的数十篇散文译文, 在译文基本没有语法错误的 情况下, 作者用负迁移理论对其进行探索, 着重从词汇和句法两个方面归纳 Chinglish 现象的主要形式，分析其原因，并提出相关对策。

关键词: Chinglish，母语负迁移，散文英译，对策 